\title{
Mesure du module en petites déformations d'un sol autour d'une conduite enterrée : présentation d'un nouvel essai in situ
}

\author{
Olivier Thépot et Roger Frank
}

\begin{abstract}
Résumé : Nous présentons dans cet article une nouvelle méthode de mesure de la déformabilité des sols encaissant des conduites enterrées qui consiste à utiliser la conduite comme une sonde pressiométrique dilatable. La dilatation de la conduite est obtenue par un essai de vérinage interne qui provoque une ovalisation tridimensionnelle relativement faible de l'ordre de $0,05 \%$. On mesure la courbe effort-déplacement dans l'axe de poussée ainsi que l'amortissement avec la distance de la déformation dans le sens longitudinal à une distance d'un diamètre. Connaissant les caractéristiques mécaniques de la conduite, on peut calculer le module en très petites déformations du sol encaissant. Cet article présente le dispositif de l'essai ainsi que la technique de calcul qui est basée sur une modélisation tridimensionnelle paramétrique par la méthode des éléments finis. Les résultats obtenus sur un élément de tuyau en fonte de $1,4 \mathrm{~m}$ de diamètre et de $8,0 \mathrm{~m}$ de longueur sont présentés. Les modules calculés sont comparés à ceux donnés par des essais en laboratoire à la colonne résonnante et au triaxial cyclique.
\end{abstract}

Mots clés : conduites enterrées, essai de vérinage, module du sol, interaction sol-structure, analyse tridimensionnelle, éléments finis.

\begin{abstract}
A new method of measurement of backfill stiffness around buried flexible pipe is presented. It consists of using flexible pipe as an expanding pressiometric probe. The pipe dilatation is obtained by an internal jacking test that causes a relatively small three-dimensional "ovalization" around $0.05 \%$. We obtain a force-displacement curve along the force axis as well as the damping of the displacement in the longitudinal direction up to one diameter in range. Knowing the pipe mechanical characteristics, we can calculate Young's modulus of the backfill in the domain of small deformations. The paper describes the test procedure as well as the modulus computation technique, which is based upon a three-dimensional parametric study using the finite element method. The results obtained in a jacking test con-ducted on iron pipe of $1.4 \mathrm{~m}$ in diameter and $8.0 \mathrm{~m}$ in length are presented. The computed moduli are compared with the ones obtained in laboratory testing using resonant-column and cyclic triaxial tests.
\end{abstract}

Key words: buried pipes, jacking test, soil stiffness, soil-structure interaction, three-dimensional analysis, finite elements.

\section{Introduction}

La déformabilité du sol encaissant des conduites enterrées revêt une importance particulière pour les conduites souples qui subissent des déformations significatives (plus de $1 \%$ du diamètre). En effet, l'interaction entre la conduite et le sol dépend surtout de la zone d'enrobage de la conduite et de la qualité de l'interface sol-conduite. Comme dans tous les problèmes d'interaction sol-structure, la rigidité relative entre la conduite et le sol et l'état de l'interface (glissante ou frottante) sont déterminants dans le bilan des sollicitations.

Les méthodes habituellement utilisées pour calculer les conduites souples utilisent des modèles à barres et ressorts

O. Thépot. Société Anonyme de Gestion des Eaux de Paris (SAGEP), 9, rue Schoelcher, 75014 Paris, France.

R. Frank. Centre d'Enseignement et de Recherche en Mécanique des Sols (ENPC-CERMES), 6 et 8, avenue Blaise Pascal, Cité Descartes, Champs-sur-Marnes, 77455 Marne-laVallée CEDEX 2, France.
(Spangler 1941). Ces méthodes « aux modules de réaction » ont permis d'obtenir des solutions analytiques pratiques pour les projeteurs, et on les retrouve naturellement dans le calcul des pieux et des écrans de soutènement. En revanche, elles souffrent d'une absence de justification théorique, car le module de réaction ne peut pas être considéré comme une caractéristique intrinsèque du sol, et il n'existe aucune méthode physique rigoureuse pour en fixer la valeur.

La méthode des éléments finis est de ce point de vue plus satisfaisante car elle modélise le sol par un continuum de manière réaliste, contrairement aux modèles à ressorts indépendants, et elle permet d'utiliser des lois de comportement non linéaires (élasto-plastiques). Dans le domaine des conduites enterrées, cette méthode a été utilisée en particulier par Abel et al. (1973), Duncan (1979) et Moore (1987a, 1987b).

Toutefois, si la méthode des éléments finis permet d'utiliser des caractéristiques intrinsèques du sol, le problème de mesurer ces caractéristiques demeure. Le choix du module d'Young pose un problème délicat, car le sol a un comportement fortement non linéaire même à l'intérieur de la surface de charge. Il décroît rapidement avec la déformation, et ce n'est que pour des déformations très faibles, 
Fig. 1. Principe de l'essai (les déformations sont très exagérées).
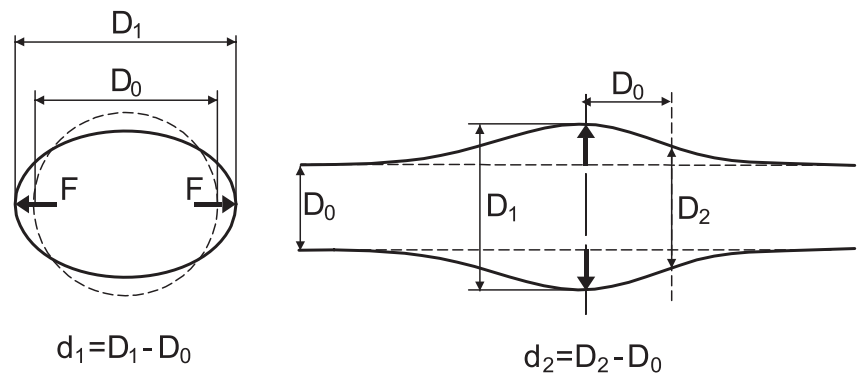

inférieures à $10^{-5}$, que l'on peut observer un comportement élastique linéaire (Hicher 1994).

De ce fait la plupart des valeurs de modules proposées par les méthodes de calculs des conduites souples sont issues de l'observation des déformations des conduites après leur remblaiement et non pas de mesures directes dans les matériaux de remblaiement. La détermination empirique des modules de sols est aussi justifiée par le fait que le module apparent du sol, tel qu'il ressort d'une analyse inverse, dépend autant de la déformabilité de la conduite et de la mise en ouvre du remblai (phasage etc.) que de ses caractéristiques intrinsèques.

L'essai que nous présentons dans la suite de cet article permet de mesurer le module de déformation du sol encaissant d'une conduite enterrée, dans une plage de déformation comprise entre $10^{-5}$ et $10^{-3}$. Ce module est particulièrement adapté à la modélisation du comportement en service d'une conduite enterrée par la méthode des éléments finis.

\section{Principe de l'essai}

L'essai consiste à ovaliser une conduite par un dispositif de vérinage interne qui exerce deux forces diamétralement opposées et à mesurer la déformation tridimensionnelle résultante. Trois mesures sont effectuées (fig. 1) :

la force exercée par le vérin, notée $F$;

l'allongement du diamètre horizontal au niveau de l'axe du vérin, noté $d_{1}=D_{1}-D_{0}$, que nous appelons 《 ovalisation principale » où $D_{0}$ est le diamètre initial (égal au diamètre intérieur plus l'épaisseur) et $D_{1}$ est le diamètre final (après application de la force);

l'allongement du diamètre horizontal à une distance d'un diamètre de l'axe du vérin, noté $d_{2}=D_{2}-D_{0}$, que nous appelons « ovalisation secondaire » où $D_{2}$ est le diamètre final;

On définit les paramètres suivants :

le taux d'ovalisation principale $O_{\mathrm{p}}=d_{1} / D_{0}$ en $\%$;

le taux d'ovalisation secondaire $O_{\mathrm{s}}=d_{2} / D_{0}$ en $\%$;

la force annulaire $F_{\mathrm{a}}=2 F / D_{0}$, rapport entre la force et le diamètre moyen multiplié par deux, en $\mathrm{MN} / \mathrm{m}$;

la raideur $R=F_{\mathrm{a}} / O_{\mathrm{p}}=2 F / d_{1}$, rapport entre la force annulaire et le taux d'ovalisation principale, en $\mathrm{MN} / \mathrm{m}$;
Fig. 2. Courbe force annulaire $F_{\mathrm{a}}$ - taux d'ovalisation principale $O_{\mathrm{p}}$.

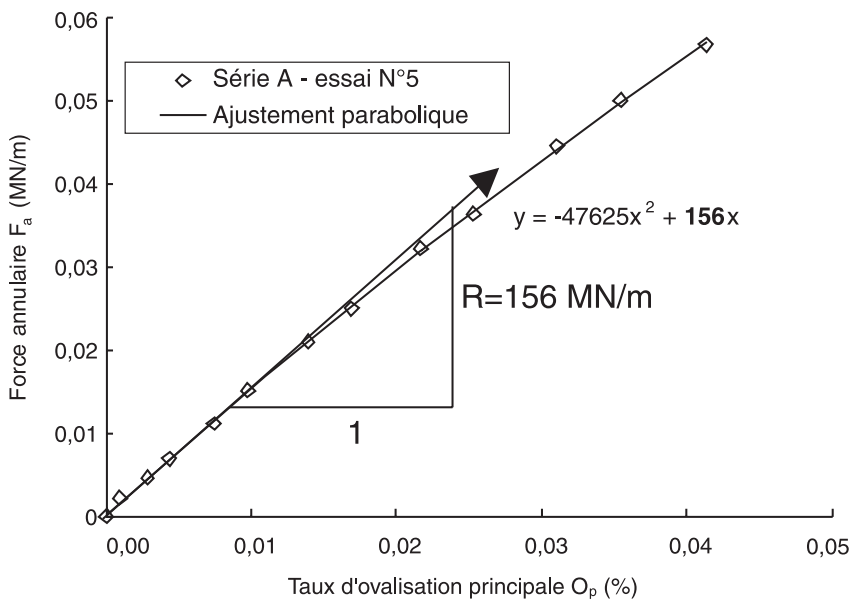

Fig. 3. Courbe taux d'ovalisation secondaire $O_{\mathrm{s}}-\operatorname{taux}$ d'ovalisation principale $O_{\mathrm{p}}$.

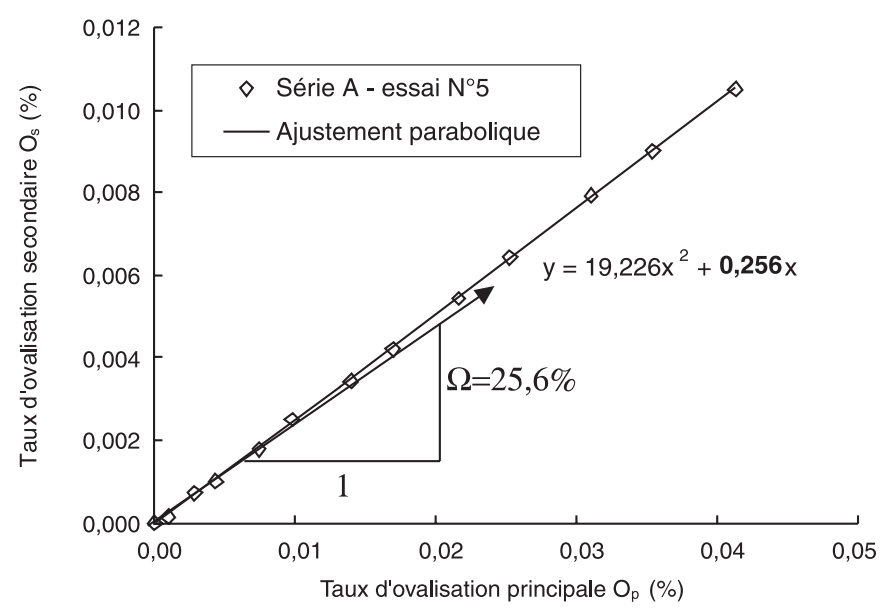

le taux de transmission $\Omega=d_{2} / d_{1}=O_{\mathrm{s}} / O_{\mathrm{p}}$ en $\%$, rapport entre l'allongement secondaire et l'allongement principal, sans dimensions.

\section{Résultats typiques}

Le résultat d'un essai est constitué de deux courbes : une courbe force annulaire - taux d'ovalisation principale ou courbe de raideur (fig. 2) et une courbe taux d'ovalisation secondaire - taux d'ovalisation principale ou courbe de transmission (fig. 3). Ces courbes ne sont généralement pas linéaires, elles présentent une certaine convexité que l'on prend en compte en ajustant un polynôme du second degré de la forme $y=a x^{2}+b x$ par la méthode des moindres carrés.

La raideur $R$ est définie comme la pente à l'origine de la courbe de raideur (fig. 2) que l'on calcule par le coefficient b du polynôme ajusté.

De même le taux de transmission $\Omega$ est défini comme la pente à l'origine de la courbe de transmission (fig. 3). 
Fig. 4. Dispositif d'essai, vue générale.

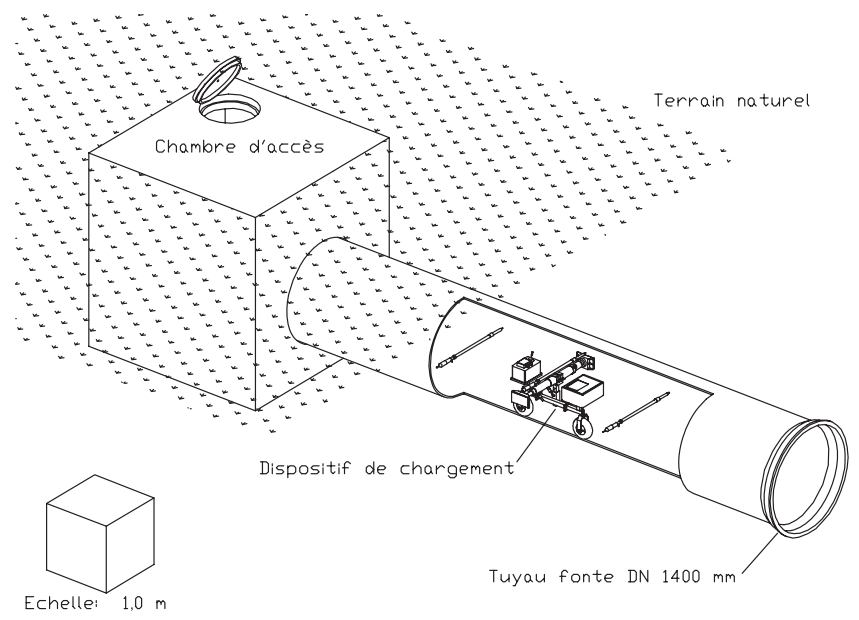

\section{Interprétation de l'essai}

Contrairement à l'essai pressiométrique, on ne dispose pas de solutions analytiques pour guider l'interprétation des résultats. En effet, la déformée tridimensionnelle de la conduite est trop complexe, et le champ de déformation dans le sol est loin d'être uniforme (il ne l'est pas non plus dans l'essai pressiométrique). La méthode des éléments finis est ici un outil indispensable, car elle permet de prendre en compte l'aspect tridimensionnel du problème. Il subsiste toutefois une difficulté qui est liée à la forte variation du module sécant du sol avec la déformation. Comme pour l'essai pressiométrique, on mesure en fait un module sécant « moyen » qui intègre les modules des différentes parties du sol qui ne sont pas au même niveau de déformation (Muir Wood 1990), on notera toutefois qu'ils suivent les mêmes chemins contraintes-déformations mais avec un décalage. En revanche, il n'y a pas, pour des raisons évidentes, de remaniement du sol à l'interface sol-conduite. On sait que le remaniement du sol est le facteur principal qui limite les mesures en très faibles déformations (Bellotti et al. 1989). Par conséquent, en l'absence de remaniement, on a intérêt à déformer le moins possible la conduite de telle manière à rester dans le domaine quasi-linéaire du sol, ce qui revient à limiter le taux d'ovalisation de la conduite à $10^{-4}$. Dans ces conditions, on mesure le module maximum du sol qui est proche du module dynamique.

Pour exprimer le module du sol encaissant en fonction des paramètres mesurés $R$ (raideur) et $\Omega$ (taux de transmission), nous conduisons une étude paramétrique par un modèle élastique linéaire aux éléments finis et l'analyse inverse.

\section{Application à une conduite en fonte de $1400 \mathrm{~mm}$ de diamètre intérieur}

Des essais d'ovalisation ont été effectués dans une conduite en fonte de $1400 \mathrm{~mm}$ de diamètre qui a été posée en tranchée dans un site expérimental situé à Champcueil dans le département de l'Essonne (France). Le sol de ce site est constitué d'un sable de Fontainebleau relativement argileux en surface.
Fig. 5. Dispositif de chargement et de mesure.

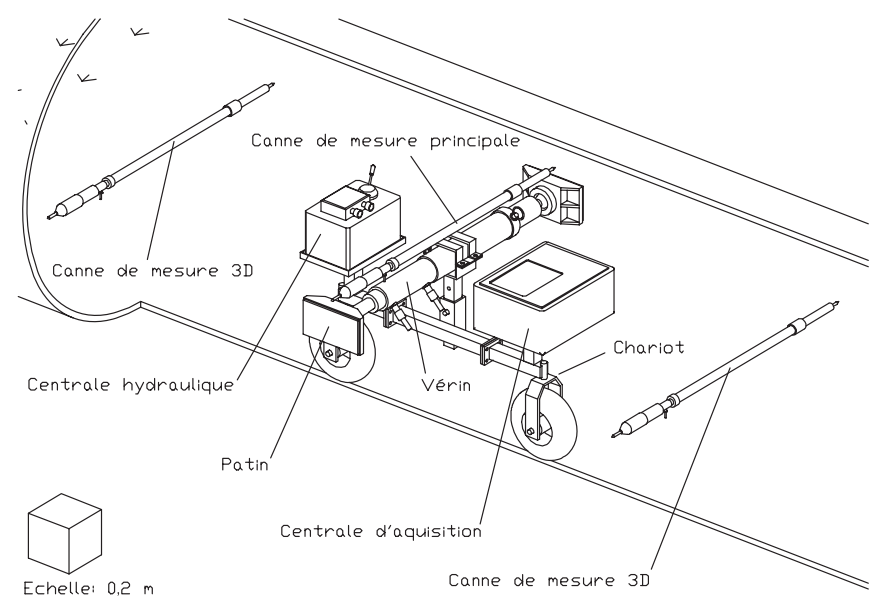

\section{Description du dispositif expérimental}

Le dispositif expérimental (fig. 4) est constitué d'une conduite en fonte de $8,16 \mathrm{~m}$ de longueur et $1,40 \mathrm{~m}$ de diamètre intérieur et d'une chambre d'accès en béton armé. La hauteur de remblai au-dessus de la buse est de 1,0 m. La conduite a été posée dans une tranchée de 2,80 $\mathrm{m}$ de largeur et $2,70 \mathrm{~m}$ de hauteur et remblayée avec le matériau du site (fig. 5).

Le dispositif de l'essai comprend (fig. 5 et 6) :

- un système de chargement mécanique constitué d'un vérin hydraulique pouvant développer des cycles d'efforts en deux points diamétralement opposés par l'intermédiaire de deux patins;

- un ensemble de capteurs de déplacements montés sur des cannes de mesure télescopiques;

- une centrale d'acquisition constituée d'un microordinateur muni d'une carte d'acquisition;

- un chariot permettant de véhiculer l'ensemble.

Le tout est à géométrie variable.

\section{Instrumentation}

L'instrumentation comprend trois cannes télescopiques disposées horizontalement (fig. 5 et 6), une canne « principale » située au-dessus du vérin et deux cannes « tridimensionnelles » disposées symétriquement à 1,40 m de l'axe du vérin. On remarquera que la canne principale ne donne pas exactement l'allongement du diamètre de la conduite dans l'axe de poussée car elle est située à $10 \mathrm{~cm}$ au-dessus de cet axe.

Les cannes tridimensionnelles permettent de caractériser l'amortissement de la déformation dans le sens longitudinal (paramètre $\Omega$ ). Nous avons utilisé deux cannes pour vérifier la symétrie de la déformation (une seule canne suffit a priori, puisque tout est symétrique par rapport à l'axe du vérin). Ces cannes télescopiques comportent des capteurs de déplacement de type LVDT (Linear Voltage Differential Transductor).

Le dispositif de chargement est constitué d'un vérin pouvant appliquer des efforts de 0 à $300 \mathrm{kN}$ et de deux patins de $28 \times 16 \mathrm{~cm}$. La fixation des patins sur le vérin est assurée par des rotules qui limitent les efforts tangentiels parasites. La mesure de l'effort est assurée par un capteur de 
Fig. 6. Vues en plan et en coupe du dispositif de chargement.
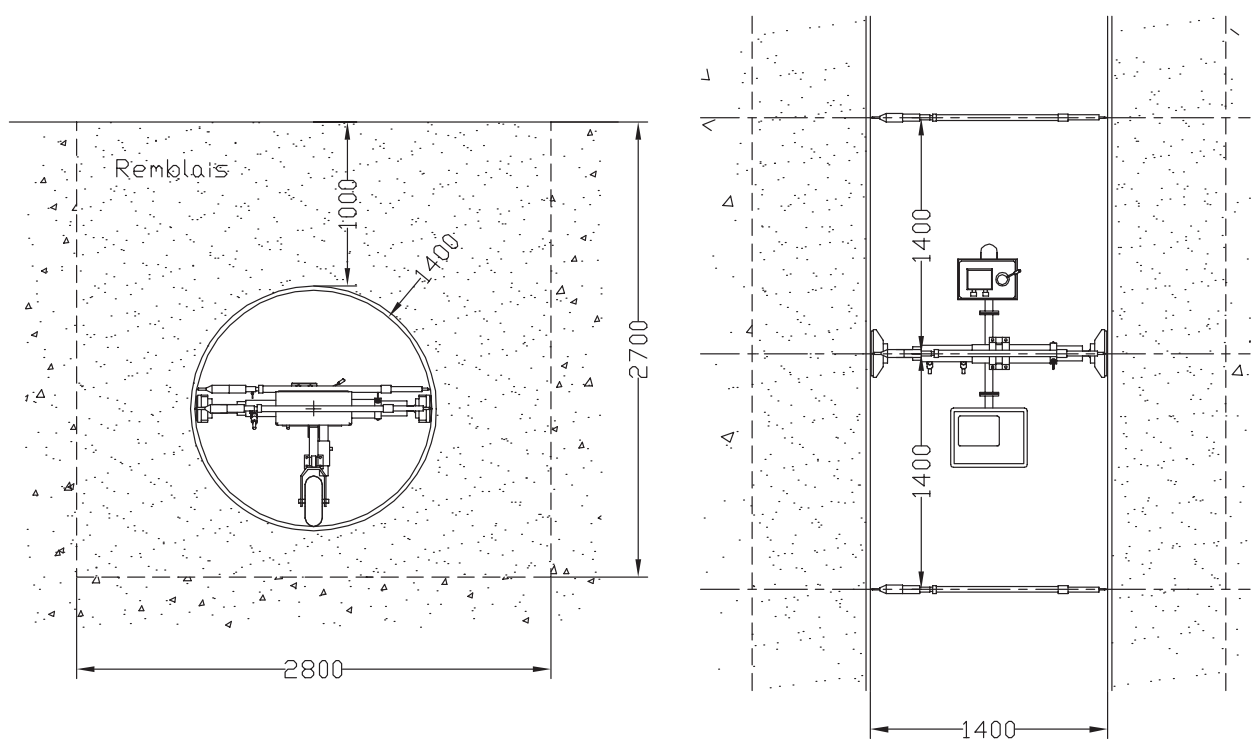

Fig. 7. Courbe de chargement-déchargement.

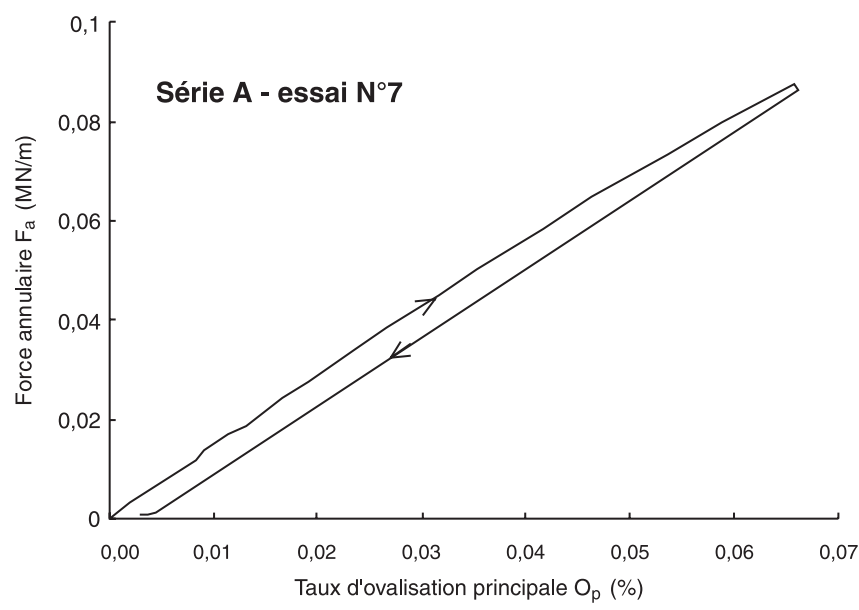

pression disposé sur le circuit d'alimentation en huile du vérin. L'ensemble vérin-patins est disposé horizontalement au milieu de la conduite à 4,08 m des extrémités

\section{Déroulement des essais}

Un essai comporte un cycle de chargement-déchargement (fig. 7). La vitesse de montée en charge est pratiquement constante $(8 \mathrm{kN} / \mathrm{s})$; en revanche le déchargement est rapide et non maîtrisé.

Un premier essai d'ovalisation a été effectué avant le remblaiement de la conduite pour mesurer ses caractéristiques propres $R_{\mathrm{c}}$ (raideur propre) et $\Omega_{\mathrm{c}}$ (taux de transmission propre).

Après remblaiement, trois séries d'essais successives A, B et $C$ (16 essais au total) ont été effectuées en augmentant progressivement le taux d'ovalisation principale $O_{\mathrm{p}}$. Les taux d'ovalisation principale maxima varient entre $0,015 \%$ et $0,24 \%$, les efforts maxima appliqués varient entre 17 et 172 $\mathrm{kN}$ (tableau 1).

\section{Résultats des essais (tableau 1)}

La raideur propre de la conduite à « l'air libre » $R_{\mathrm{c}}$ est de $28,1 \mathrm{MN} / \mathrm{m}$ et le taux de transmission propre $\Omega_{\mathrm{c}}$ est de $71,9 \%$. Ce taux est très élevé et il est encore supérieur à $30 \%$ à l'extrémité de la conduite (soit à près de trois diamètres de distance du vérin). On remarquera que le taux à gauche $(75,0 \%)$ est sensiblement différent du taux à droite $(68,8 \%)$; cet effet est dû à la présence de l'emboîtement à gauche qui rigidifie la conduite (fig. 4).

La conduite enterrée sous $1 \mathrm{~m}$ de remblai est cinq fois plus raide $(R=152 \mathrm{MN} / \mathrm{m}$ en moyenne sur la série A). Le taux de transmission est divisé par $3(\Omega=24,9 \%$ en moyenne). Le remblai modifie donc de manière très sensible le comportement de la conduite, ce qui est une surprise, car on néglige habituellement l'interaction sol-conduite pour des faibles profondeurs. La dissymétrie des taux de transmission à gauche et à droite a presque disparu, cachée par la raideur (symétrique) apportée par le remblai. Le taux de transmission est en quelque sorte une mesure directe de la raideur relative entre la conduite et le sol ou de l'intensité de l'interaction sol-conduite.

Les courbes ne sont plus linéaires, ce qui n'est pas étonnant, puisque la raideur du sol dépend de la déformation. On constate aussi sur l'ensemble des trois séries d'essais que la raideur décroît progressivement. Il est probable que la raideur se stabiliserait après un nombre important de cycles d'essais (phénomène d'accommodation) mais nous avons volontairement limité l'objectif de cette expérimentation à l'étude de la réponse du sol au premier cycle de chargement.

\section{Interprétation des résultats : Calcul du module du sol encaissant}

On considère une conduite circulaire d'épaisseur $e$, de diamètre $D_{0}$ constituée d'un matériau homogène élastique linéaire de module $E_{\mathrm{c}}$, dans un massif élastique linéaire homogène isotrope infini de module $E_{\mathrm{s}}$. Le coefficient de Poisson du sol est fixé initialement à 0,33 et celui de la 
Tableau 1. Résultats des essais de vérinage interne.

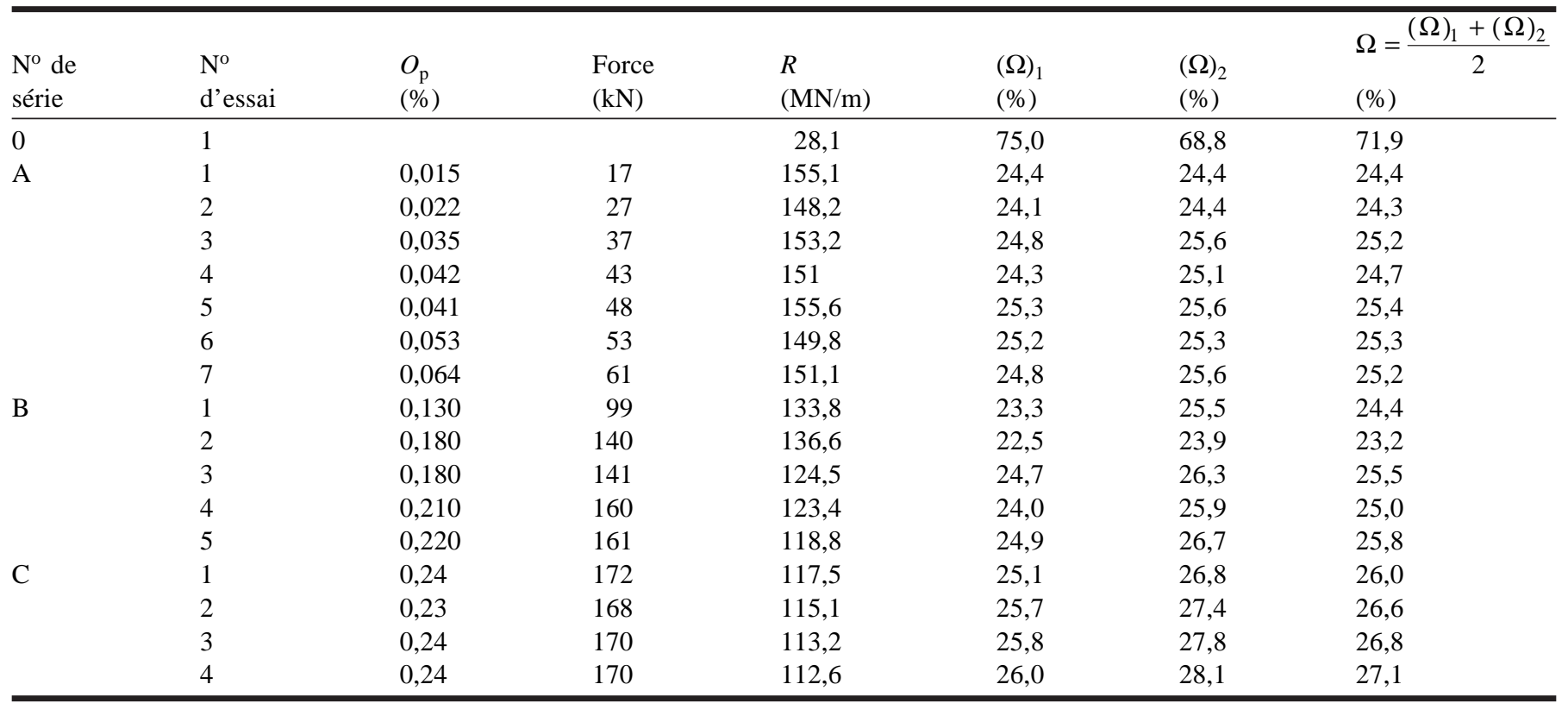

Nota : $(\Omega)_{1}$, taux de transmission mesuré par la canne gauche; $(\Omega)_{2}$, taux de transmission mesuré par la canne droite.

Fig. 8. Dessin du maillage (logiciel CESAR).

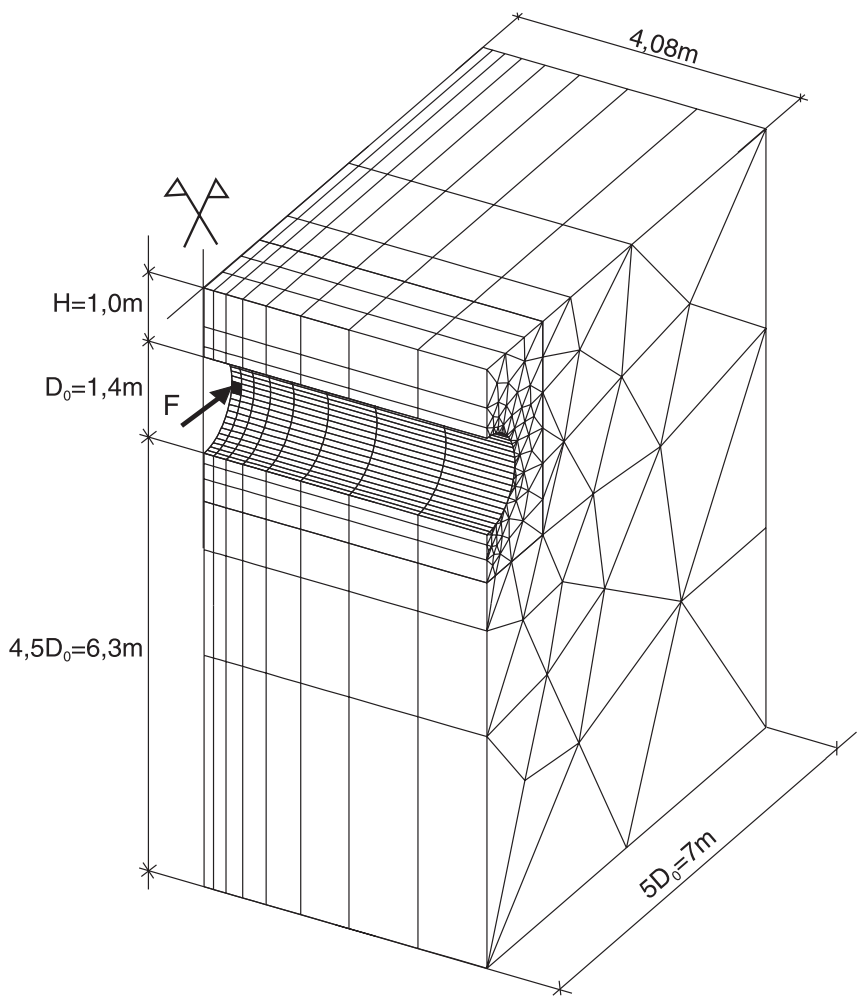

conduite à 0,25 . Le modèle paramétrique ainsi défini comporte quatre paramètres.

La raideur $R$ et le taux de transmission $\Omega$ sont fonctions des caractéristiques mécaniques du massif $\left(E_{\mathrm{s}}\right)$ et de celles de la conduite $\left(e, D, E_{\mathrm{c}}\right)$. Inversement si l'on connaît les caractéristiques mécaniques de la conduite, il est possible de calculer le module du sol en fonction de la raideur $R$ ou du taux de transmission $\Omega$.

Le calcul du module du sol encaissant ne peut pas se faire par une méthode analytique à cause de l'aspect tridimensionnel $\mathrm{du}$ problème. Nous utilisons donc la méthode des éléments finis en élasticité linéaire qui permet de prendre en compte la géométrie exacte du problème. Une fois le modèle construit, il suffit de faire varier le module du sol dans une plage suffisamment large, de 0 à $200 \mathrm{MPa}$ par exemple.

Dans un premier temps nous avons modélisé la conduite seule, car il y avait une difficulté liée à la présence sur la face interne d'un revêtement à base de ciment. La paroi de la conduite est en effet constituée de l'extérieur vers l'intérieur, de $17 \mathrm{~mm}$ de fonte ductile et $9 \mathrm{~mm}$ d'un mortier à base de ciment. Ce mortier donne à la conduite une surface d'écoulement lisse et protège la fonte $\mathrm{du}$ risque d'incrustation. Compte tenu de la taille du modèle final (conduite + sol), il n'était pas possible de modéliser le revêtement interne; il aurait fallu pour cela traiter la conduite avec des éléments de massifs avec au minimum quatre éléments dans l'épaisseur. Nous avons donc déterminé une épaisseur de fonte équivalente au bi-couche fonte + ciment en comparant les déformations d'un modèle en éléments de massif à celui d'un modèle simplifié en éléments de coque. L'épaisseur équivalente obtenue est de $21 \mathrm{~mm}$ avec le module de la fonte.

\section{Description du modèle aux éléments finis}

Il s'agit d'un modèle tridimensionnel en élasticité linéaire. Les symétries mécaniques et géométriques permettent de ne traiter que le quart du modèle physique (fig. 8). Le modèle est étendu à une distance de l'ordre de cinq diamètres de l'axe de la conduite (sous la conduite et sur les côtés). Les calculs ont été réalisés à l'aide du code CESAR du Laboratoire Central des Ponts et Chaussées (Humbert 1989). 
Tableau 2. Résultats des calculs CESAR.

\begin{tabular}{ccll}
\hline $\begin{array}{l}\text { Module du sol } \\
E_{\mathrm{s}}(\mathrm{MPa})\end{array}$ & $\begin{array}{l}\text { Raideur } R \\
(\mathrm{MN} / \mathrm{m})\end{array}$ & $\begin{array}{l}\text { Taux de } \\
\text { transmission } \Omega(\%)\end{array}$ & $\begin{array}{l}R \times \Omega^{1,2} \\
(\mathrm{MN} / \mathrm{m})\end{array}$ \\
\hline 0,1 & 32 & 72,5 & 21,8 \\
10 & 59 & 52,9 & 27,5 \\
25 & 85 & 40,7 & 28,9 \\
50 & 117 & 31,2 & 28,9 \\
100 & 168 & 22,5 & 28 \\
200 & 250 & 15,4 & 26,5 \\
\hline
\end{tabular}

La densité du maillage est adaptée à l'aspect ponctuel du chargement. La conduite comporte 28 éléments dans sa demi-périphérie et 8 éléments dans le sens longitudinal sur une demi-longueur (fig. 8). L'effort exercé par les patins est représenté par une pression uniforme qui s'exerce sur 4 éléments (en fait 2 éléments du fait de la symétrie). Le maillage illustré sur la figure 8 comporte 4381 noeuds et 1584 éléments répartis en trois groupes, il représente le quart de la conduite et le quart du massif de sol. La conduite est traitée avec des éléments de coques minces à 8 noeuds (COQ8), le sol avec des éléments pentaèdriques à 15 noeuds (MTP15).

Six calculs ont été effectués pour les modules de sol variant de 0,1 à $200 \mathrm{MPa}$. Les caractéristiques mécaniques des matériaux sont les suivantes :

Conduite

$$
\begin{aligned}
& \text { Épaisseur équivalente }: e=21 \mathrm{~mm} \\
& \text { Diamètre moyen }: D_{\mathrm{o}}=1400 \mathrm{~mm} \\
& \text { Module d'Young : } E_{\mathrm{c}}=170000 \mathrm{MPa} \\
& \text { Coeficient de Poisson }: 0,25
\end{aligned}
$$

Sol

Module d'Young : $E_{\mathrm{s}}=0,1 ; 10 ; 25 ; 50 ; 100$; $200 \mathrm{MPa}$

$$
\text { Coeficient de Poisson : 0,33 }
$$

On constate (tableau 2 et fig. 9) que la raideur augmente rapidement avec le module du sol et qu'elle devient nettement supérieure à la raideur propre de la conduite. Inversement, le taux de transmission chute tout aussi rapidement. Pour un module de sol de $50 \mathrm{MPa}$, la raideur initiale est multipliée par 3,7 et le taux de transmission est divisé par 2,3 par rapport aux valeurs calculées avec $E_{\mathrm{s}}=$ $0,1 \mathrm{MPa}$.

On peut remarquer que le produit de la raideur par le taux de transmission élevé à la puissance 1,2 est quasiment constant dès que le module du sol est supérieur à quelques MPa. C'est-à-dire que l'on peut écrire avec une bonne approximation (sauf pour $E_{\mathrm{s}}=0,1 \mathrm{MPa}$ ) :

$$
R \times \Omega^{1,2} \approx 28_{\mathrm{MN} / \mathrm{m}}
$$

Cette relation empirique, très simple au demeurant, traduit le couplage qui existe entre les paramètres $R$ et $\Omega$.

Le module du sol peut s'exprimer aussi bien en fonction de la raideur $R$ que du taux de transmission $\Omega$. Afin de proposer des expressions analytiques simples, nous avons ajusté sur les cinq calculs CESAR, par la méthode des moindres
Fig. 9. Évolution de la raideur $R$ et du taux de transmission $\Omega$ en fonction du module du sol $E_{\mathrm{s}}$ (résultats CESAR).

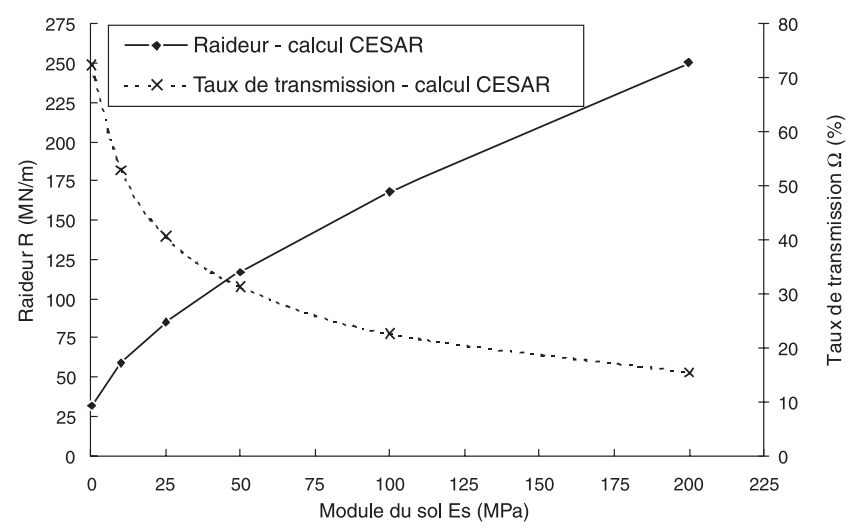

carrés, des fonctions polynomiales du deuxième degré. Au préalable, on définit un module du sol « réduit » en divisant $E_{\mathrm{s}}$ par la raideur propre de la conduite $R_{\mathrm{c}}$ et en le multipliant par le diamètre moyen $D_{0}$. De la même manière on définit une raideur « réduite » en divisant $R$ par la raideur propre $R_{\mathrm{c}}$ et en soustrayant 1 pour la centrer.

On rappelle que $R_{\mathrm{c}}$ et $\Omega_{\mathrm{c}}$ sont respectivement la raideur et le taux de transmission de la conduite à l'air libre, c'est-àdire pour un module du sol égal à zéro.

Module réduit :

$$
E_{\mathrm{s}}^{\prime}=\frac{E_{\mathrm{s}} \times D_{0}}{R_{\mathrm{c}}}
$$

Raideur réduite :

[3] $\quad R^{\prime}=\frac{R}{R_{\mathrm{c}}}-1$

On définit le facteur d'amortissement :

[4] $A=\frac{\Omega_{\mathrm{c}}}{\Omega}-1$

comme l'inverse du taux de transmission réduit moins 1 .

On obtient par ajustement sur les cinq calculs CESAR les expressions suivantes (fig. 10 et 11) :

Module réduit du sol en fonction de la raideur réduite :

$$
E_{\mathrm{S}}^{\prime}\left(R^{\prime}\right)=0,11 \times R^{\prime}\left(R^{\prime}+4,71\right)
$$

Module du sol en fonction de la raideur réduite et de la raideur propre de la conduite :

$$
E_{\mathrm{s}}\left(R^{\prime}\right)=0,11 \times R^{\prime}\left(R^{\prime}+4,71\right) \frac{R_{\mathrm{c}}}{D_{0}}
$$

Module réduit $\mathrm{du}$ sol en fonction $\mathrm{du}$ facteur d'amortissement :

$[6 a]$

$$
E_{\mathrm{S}}^{\prime}(A)=0,3 \times A \times(A+4,29)
$$

Module du sol en fonction du facteur d'amortissement et de la raideur propre de la conduite : 
Tableau 3. Résultats des calculs, module du sol encaissant.

\begin{tabular}{|c|c|c|c|c|c|c|}
\hline $\begin{array}{l}\mathrm{N}^{\mathrm{o}} \text { de } \\
\text { série }\end{array}$ & $\begin{array}{l}\mathrm{N}^{\mathrm{o}} \\
\text { d'essai }\end{array}$ & $\begin{array}{l}R \times \Omega^{1,2} \\
(\mathrm{MN} / \mathrm{m})\end{array}$ & $\begin{array}{l}\text { Raideur réduite } \\
R^{\prime}=\frac{R}{28,1}-1\end{array}$ & $\begin{array}{l}\text { Module du } \\
\text { sol } E_{\mathrm{s}}\left(R^{\prime}\right) \\
(\mathrm{MPa})\end{array}$ & $\begin{array}{l}\text { Amortissement } \\
A=\frac{71,9 \%}{\Omega}-1\end{array}$ & $\begin{array}{l}\text { Module du sol } \\
E_{\mathrm{s}}(A)(\mathrm{MPa})\end{array}$ \\
\hline \multirow[t]{4}{*}{ A } & 1 & 28,5 & 4,52 & 92 & 1,95 & 73 \\
\hline & 3 & 29,3 & 4,45 & 90 & 1,85 & 68 \\
\hline & 4 & 28,2 & 4,37 & 88 & 1,91 & 71 \\
\hline & 7 & 28,9 & 4,38 & 88 & 1,85 & 68 \\
\hline \multirow[t]{5}{*}{ B } & 1 & 24,6 & 3,76 & 70 & 1,95 & 73 \\
\hline & 2 & 23,7 & 3,86 & 73 & 2,1 & 81 \\
\hline & 3 & 24,2 & 3,43 & 62 & 1,82 & 67 \\
\hline & 4 & 23,3 & 3,39 & 61 & 1,88 & 70 \\
\hline & 5 & 23,4 & 3,23 & 57 & 1,79 & 66 \\
\hline $\mathrm{C}$ & 4 & 23,3 & 3,0 & 51 & 1,65 & 59 \\
\hline
\end{tabular}

Fig. 10. Module réduit du sol $E_{\mathrm{s}}^{\prime}$ fonction de la raideur réduite $R^{\prime}$, ajustement parabolique sur des résultats CESAR.

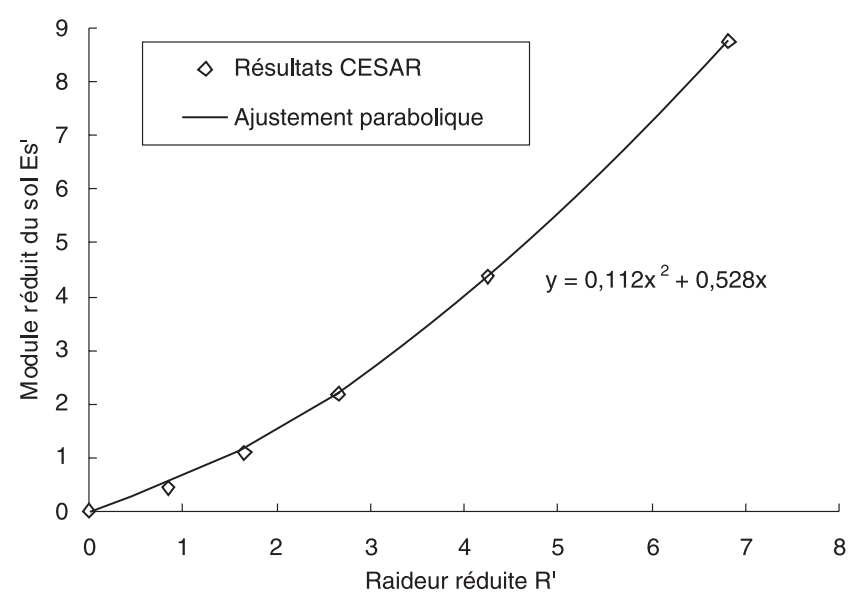

$[6 b]$

$$
E_{\mathrm{s}}(A)=0,3 \times A \times(A+4,29) \frac{R_{\mathrm{c}}}{D_{0}}
$$

Le tableau 3 donne les modules de sol calculés avec les formules précédentes. Pour la première série d'essai, le module moyen issu du calcul par la raideur réduite est de $89 \mathrm{MPa}$, et celui issu du calcul par le facteur d'amortissement est de $70 \mathrm{MPa}$. Le produit $R \times \Omega^{1,2}$ est pratiquement égal à la valeur prévue par l'approximation donnée par [1] (28 MN/m). On peut donc retenir un module moyen de l'ordre de $80 \mathrm{MPa}$ (moyenne des deux approches). Pour les deux séries suivantes, le module issu du calcul par la raideur devient plus faible que celui issu du calcul par le facteur d'amortissement. De même le produit $R \times \Omega^{1,2}$ diminue. Il est probable que l'hypothèse de linéarité n'est plus vérifiée, l'application de déformations croissantes dans le sol provoque une diminution du module maximum et une anisotropie induite. Ce phénomène est connu : on peut ob-
Fig. 11. Module réduit du sol $E_{\mathrm{s}}^{\prime}$ fonction du facteur d'amortissement $A$, ajustement parabolique sur des résultats CESAR.

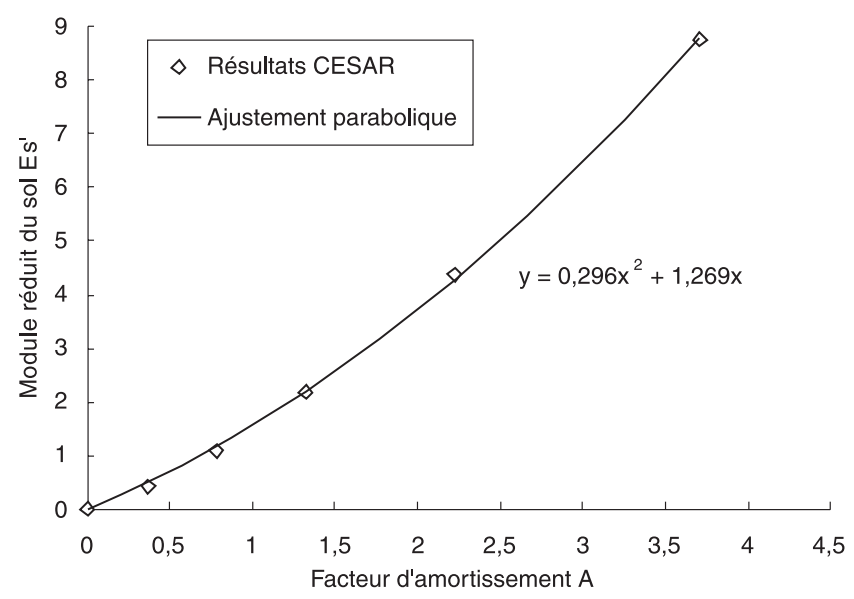

server pour certains sols, argiles ou matériaux calcaires, une diminution du module maximum après un premier chargement (par exemple, Lo Presti et al. 1993).

\section{Résultats des essais in situ et en laboratoire sur le sol encaissant}

Afin de caractériser la déformabilité du sol, des essais de laboratoire à la colonne résonnante et au triaxial cyclique et un essai « cross-hole » in situ ont été effectués pour mesurer le module maximum du sol intact. Les essais en laboratoire avait pour but d'explorer une plus large gamme de sollicitation que l'essai cross-hole (dont le domaine de déformation ne dépasse pas $10^{-6}$ ) et de vérifier l'effet du remaniement. La colonne résonnante permet d'explorer la gamme de déformation comprise entre $10^{-5}$ et $5 \times 10^{-4}$, le triaxial cyclique permet de compléter la courbe jusqu'au pic de rupture. 
Fig. 12. Courbe en S, évolution du module d'Young du sol $E_{\mathrm{s}}$ en fonction de la dvéformation de cisaillement $\gamma$.

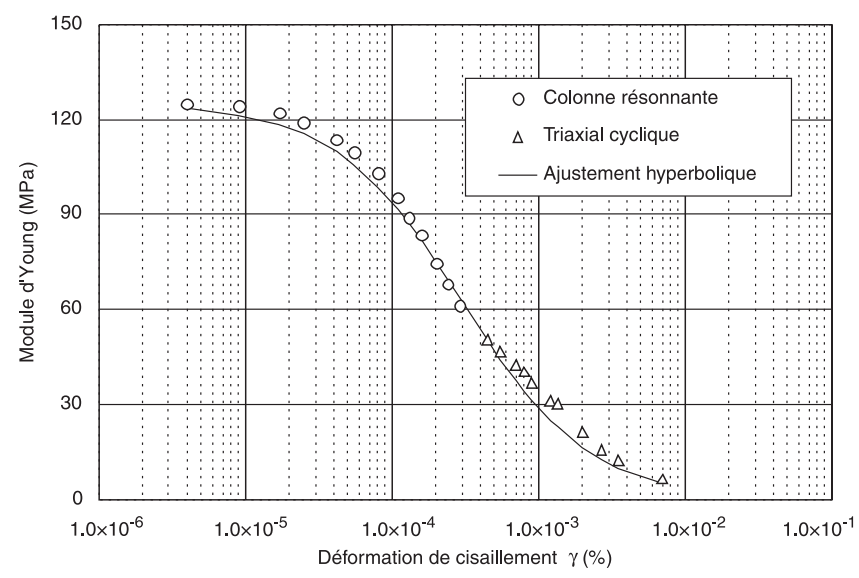

L'échantillon de sol testé a été reconsolidé à une pression de confinement de $50 \mathrm{kPa}$ qui correspond à une profondeur de l'ordre de $3 \mathrm{~m}$, un peu supérieure à la profondeur moyenne de la tranchée qui est plutôt de $2 \mathrm{~m}$. Les données issues des deux essais (colonne résonnante et triaxial) se « raccordent » plutôt bien autour d'une déformation de $5 \times$ $10^{-4}$. Dans un diagramme semi-logarithmique, la courbe module sécant-déformation a typiquement une forme en $\mathrm{S}$ (fig. 12) avec au début un plateau jusqu'à une déformation inférieure à $10^{-5}$ puis une chute brutale jusqu'à $10^{-2}$ où le sol est complètement «plastifié ». En dehors du domaine élastique, dont la plage est très faible $\left(\varepsilon<10^{-5}\right)$, des déformations irréversibles se développent, et la relation contrainte-déformation devient rapidement non linéaire. Le module sécant est alors une fonction décroissante de la déformation, et de nombreux auteurs ont cherché à modéliser la relation module-déformation par une fonction mathématique simple (Hardin et Drnevich 1972; Seed et Idriss 1970).

Hardin et Drnevitch ont proposé en 1972 une fonction hyperbolique à deux paramètres sous la forme :

$$
\frac{G_{\mathrm{s}}}{G_{\mathrm{Max}}}=\frac{1}{1+\frac{\gamma}{\gamma_{\mathrm{r}}}}
$$

avec :

$$
\begin{aligned}
& G_{\mathrm{s}}=\text { module de cisaillement sécant } \\
& G_{\mathrm{Max}}=\text { module de cisaillement maximal } \\
& \gamma=\text { déformation de cisaillement } \\
& \gamma_{\mathrm{r}}=\text { déformation de référence égale à } \tau_{\mathrm{Max}} / G_{\mathrm{Max}} \\
& \text { ou } \tau_{\mathrm{Max}} \text { désigne la contrainte de cisaillement } \\
& \text { maximale (rupture). }
\end{aligned}
$$

Dans le cas présent nous avons simplement ajusté une loi hyperbolique par une méthode de moindres carrés (sans nous soucier de la contrainte de cisaillement maximale). On obtient la relation suivante :
[8]

$$
\frac{E_{\mathrm{S}}}{E_{\mathrm{Max}}}=\frac{1}{1+\frac{\gamma}{3 \times 10^{-4}}}
$$

avec $E_{\mathrm{Max}}=125 \mathrm{MPa}$

Cette relation montre que le module sécant est divisé par 2 quand la déformation atteint $3 \times 10^{-4}$ et par 4,3 pour une déformation de $10^{-3}(0,1 \%)$.

Un essai « cross-hole » a été réalisé entre deux forages de $6,5 \mathrm{~m}$ de profondeur et distants de $6 \mathrm{~m}$. Une mesure a été prise à tous les mètres à partir de $1 \mathrm{~m}$ de profondeur. On a constaté un gradient important du module avec la profondeur, qui est dû au fait que l'on passe d'un sable fortement argileux en surface à un sable propre à partir de $6 \mathrm{~m}$ de profondeur. On peut retenir pour les 3 premiers mètres un module en très faibles déformations de l'ordre de $270 \mathrm{MPa}$ qui est nettement plus élevé que le module maximum de $125 \mathrm{MPa}$ mesuré à la colonne résonnante (qui correspond à une profondeur de $3 \mathrm{~m}$ ). Il nous semble évident que cet écart est dû à l'effet du remaniement qui est particulièrement important pour les sols argileux.

Les mesures à la colonne résonnante sont donc représentatives non pas de la raideur du sol en place, mais plutôt de celle du sol mis en remblai autour de la conduite qui a été complètement remanié et recompacté artificiellement. Si l'on compare maintenant le module calculé (80 MPa) au module maximum mesuré dans l'essai de colonne résonnante $(125 \mathrm{MPa})$ ou à la courbe en $\mathrm{S}$, on peut dire que l'ordre de grandeur est respecté. En effet, l'essai d'ovalisation donne le module moyen du remblai sur une hauteur de l'ordre du diamètre de la conduite. Il est donc forcément inférieur au module du remblai à sa base. Sur la courbe en $\mathrm{S}$, $80 \mathrm{MPa}$ correspond à une déformation de $1,7 \times 10^{-4}$ $(0,017 \%)$. Ceci dit, il faut se garder de faire des comparaisons directes entre des mesures sur échantillons homogènes et le résultat d'un essai in situ qui crée dans le sol un champ de déformation très complexe.

\section{Conclusions}

Un nouvel essai in situ a été présenté qui permet de mesurer le module en petites déformations $\left(<10^{-3}\right) \mathrm{du}$ sol autour d'une canalisation flexible. L'interprétation de l'essai utilise un modèle paramétrique aux éléments finis et une technique de calage inverse. Les résultats obtenus sur le remblai d'une canalisation faiblement enterrée ont été comparés à des essais de laboratoire à la colonne résonnante et au triaxial cyclique. Un bon accord entre le module calculé par calage inverse et le module maximum mesuré à la colonne résonnante a été obtenu.

Plusieurs applications sont possibles pour cet essai :

La première concerne l'étude du comportement en service des conduites enterrées (collecteurs, galeries, etc.) qui nécessite de connaître le module du sol encaissant dans le domaine des déformations inférieures à $0,1 \%$.

La seconde application concerne la vérification du compactage des remblais pour les conduites posées en tranchées. Cette vérification revêt une importance particulière pour les conduites souples qui subissent des déformations significatives et nécessitent de ce fait une zone de remblai soigné enrobant la canalisation. 
L'intérêt de cet essai est aussi qu'il montre directement l'importance de l'interaction sol-structure dans le cas d'une canalisation enterrée.

\section{Remerciements}

Les appareillages utilisés et l'expérimentation décrite dans cet article ont été entièrement financés par la Société Anonyme de Gestion des Eaux de Paris (SAGEP).

\section{Références bibliographiques}

Abel, J.F., Mark, R., et Richards, R. 1973. Stress around flexible elliptical pipes. Journal of the Soil Mechanics and Foundation Division, ASCE, 99(7) : 509-526.

Bellotti, R., Ghionna, V., Jamiolkowski, M., Rrobertson, P.K., et Peterson, R.W. 1989. Interpretation of moduli from self-boring pressuremeter tests in sand. Géotechnique, 39(2) : 269-292.

Duncan, J.M. 1979. Behaviour and design of long-span metal culverts. Journal of Geotechnical Engineering, ASCE, 105(3) : 399-418.
Hardin, B.O., et Drnevich, V.P. 1972. Shear modulus and damping in soils. Journal of the Geotechnical Engineering, ASCE, 98(6) : 603-624.

Hicher, P.Y. 1994. Elastic properties of soils. Journal of the Geotechnical Engineering, ASCE, 122(8) : 641-648.

Humbert, P. 1989. CESAR-LCPC, un code général de calcul par éléments finis. Bulletin de Liason des Laboratoires des Ponts et Chaussées. 160 : 112-116.

Lo Presti, D.C.F., Pallara, O., Lancellota, R., Armandi, M., et Maniscalco, R. 1993. Monotonic and cyclic loading behavior of two sands at small strains. Geotechnical Testing Journal, 16(4) : 409-424.

Moore, I.D. 1987a. Response of buried cylinders to surface loads. Journal of the Geotechnical Engineering, ASCE, 113(7) : 758773.

Moore, I.D. 1987b. The elastic stability of shallow buried tubes. Géotechnique, 37(2) : 151-161.

Muir Wood, D. 1990. Strain-dependent moduli and pressuremeter tests. Géotechnique, 40(3) : 509-512.

Seed, H.B., et Idriss, I.M. 1970. Soil moduli and damping factors for dynamic response analysis. Rapport $n^{\circ}$ EERC 70-10, Earthquake Engineering Researh Center, University of California, Berkeley, Calif.

Spangler, M.G. 1941. The structural design of flexible pipe culverts. The Iowa State College Bulletin, 40(30). 